\title{
Gratitud en la Psicoterapia Cognitiva: elementos para su inclusión
}

Lic. Natalia C. Moyano

\section{Resumen}

La gratitud es un estado afectivo cognitivo caracterizado por el reconocimiento de haber recibido un beneficio de parte de un agente externo de manera solidaria y gratuita. A pesar de haberse recabado numerosos datos sobre la asociación de la gratitud con el bienestar subjetivo, psicológico, salud física y capacidad de adaptación a eventos estresantes, avalando su relevancia para los programas psicoterapéuticos, preventivos y promotores de salud mental, es un constructo poco considerado a nivel académico y en su interrelación con los instrumentos psicoterapéuticos.

En el presente, se revisan los principales concepto y datos proporcionados por las investigaciones asociadas a la temática con el objetivo de analizar las posibles vías de inclusión de la gratitud dentro de la Psicoterapia Cognitiva.

Palabras clave: Gratitud - Psicoterapia Cognitiva. 


\section{Gratitude in cognitive psychotherapy}

\section{Abstract}

Gratitude is a cognitive-affective state caused by the recognition that one has received a benefit from an external agent, due to the good intentions of this agent. Despite the evidence that associate gratitude with subjective well being, psychological well being, physical health and copping with stressful events, it is not enough taken in consideration in an academic level and in its interaction with psychotherapy instruments as well.

In this article, the central concepts and information provided by the research are revised, intending to analyze possible ways to include gratitude into Cognitive Psychotherapy.

Key words: Gratitude - Cognitive Psychotherapy. 


\section{Desarrollo}

La gratitud como constructo psicológico comenzó a ser estudiada a finales de la década del 90 en el seno de la naciente Psicología Positiva (Emmons, 2007), en la cual se propone el estudio científico de aquellos elementos que posibilitan el desarrollo y crecimiento del ser humano (Seligman 2003). Seligman y Peterson (2004) encuadran la gratitud dentro de su inventario de fortalezas humanas, e invitan a prestigiosos psicólogos contemporáneos a investigar sobre las diferentes fuerzas humanas. El Dr. Robert Emmons es quien asume el desarrollo de las investigaciones sobre la temática, siendo junto a Martin McCullough el principal teórico e investigador en el área.

Emmons, McCullough y otros (McCullough et al., 2001; McCullough et al., 2002; Emmons y McCullough, 2003; McCullough et al., 2004) entiende por gratitud al estado afectivo cognitivo resultante de la percepción de haber sido beneficiado por un agente externo, de manera solidaria, desinteresada y gratuita.

El aspecto cognitivo de la gratitud alude a un reconocimiento (Emmons, 2007): el sujeto que siente agradecimiento interpreta que ha recibido algo, caracterizado a su vez como valorado social o subjetivamente. Un tercer aspecto cognitivo de la gratitud, es que el individuo debe asumir para desarrollar las emociones placenteras concomitantes que el beneficio recibido proviene de un agente externo a sí mismo (Bono y McCullough, 2006), entendido de manera amplia: puede tratarse de una persona del entorno próximo al individuo que siente agradecimiento, una persona no conocida, entes impersonales como Dios o la naturaleza (Seligman, 2003), incluso es posible vivenciar a la vida misma como benefactor. Emmons (2007) asevera que la experiencia de agradecimiento se ve potenciada si el agente benefactor es asumido como valorado, y que el hecho de realizar un beneficio ha sido para este un acto gratuito, no recompensado, desinteresado. Quien se siente agradecido asume que podría no haber sido objeto del beneficio percibido, aún cuando exista la creencia que se le debía. Por ejemplo, un trabajador agradecido puede sentir gratitud por recibir su remuneración a tiempo, a pesar de ser consciente que es su derecho y que el empleador debe cumplir con tal término; lo que determinaría la experiencia de agradecimiento es la creencia que, a pesar de deber, su empleador podría haber decidido no cumplir con tal acuerdo.

La experiencia de gratitud es una emoción placentera (Emmons, 2007); en sus manifestaciones más significativas puede alcanzar niveles elevados de intensidad, llevando a quien la experimenta hasta las lágrimas de alegría y despertando en el individuo una sensación de apreciación de la vida y satisfacción con el pasado (Seligman, 2003).

Seligman agrega a la definición de gratitud un tercer aspecto: el conductual. Para Seligman (2003), el acto de dar gracias, de expresar externamente el 
agradecimiento, forma parte del concepto de gratitud: no se puede clasificar como agradecida a una persona que no se toma el tiempo para manifestarlo externamente. Este es un punto no aclarado en relación a la temática: el mero hecho de agradecer verbalmente o con conductas de contra beneficio (devolver el favor) no implica la experimentación de las sensaciones y cogniciones específicas de la gratitud y, concomitantemente, no se posee evidencia de que quien las experimenta tienda necesariamente a manifestarlas externamente (Moyano, 2009). Al respecto, como se desplegará más adelante, ejercicios de gratitud han permitido observar que la asunción de la tarea de agradecer externamente a alguien (Emmons y McCullough, 2003; Seligman, 2003; Sheldon y Lyubomirsky, 2006) puede despertar las cogniciones y emociones propias, sugiriendo que la estrecha interrelación afecto-cognición-conducta es aplicable a la gratitud.

\section{Los niveles de la Gratitud}

Siguiendo a Rosemberg (1998), McCullough et al. (2004) clasifican la gratitud en los tres niveles que asumen los estados afectivos; esto es: rasgo afectivo, estado afectivo intermedio o humor, y emoción. Los tres niveles se relacionan entre sí a manera de cadena, por lo que el rasgo afectivo predispone a desarrollar el humor específico que, a su vez, facilita la percepción de la emoción concomitante como reacción ante un evento específico.

La gratitud, en tanto rasgo afectivo, se relaciona con rasgos de personalidad y predispone al individuo a sentir agradecimiento independientemente de los acontecimientos vitales que puedan producirla o no (McCullough et al., 2004). En general, las investigaciones que se han efectuado sobre la temática se posicionan en este nivel, que es dado en llamar habitualmente como Gratitud Disposicional. Según los autores de referencia (McCullough et al., 2002), el cuestionario de gratitud CG-6, mayormente utilizado en la investigación, es un instrumento construido y validado para medir la disposición que un individuo posee a sentir agradecimiento.

McCullough et al. (2002) señalan que la gratitud disposicional es un constructo unidimensional pero que pueden clasificarse cuatro elementos o facetas que, si bien son claramente diferenciales entre sí, no constituyen dimensiones.

La frecuencia hace referencia a la periodicidad con que un individuo percibe emociones de agradecimiento: una persona con elevada gratitud disposicional tenderá a sentirse agradecido con una elevada frecuencia. Otra faceta de consideración, es la intensidad con que es percibida la emoción, a mayor rasgo disposicional se espera que la emoción percibida sea más intensa y significativa.

La amplitud o spam refiere a la diversidad de aspectos positivos que pueden ser reconocidos y valorados como dignos de ser objeto para dar gracias: una 
persona agradecida sentirá gratitud por los favores recibidos, como por su salud, los logros conseguidos, los objetos materiales que haya logrado recabar en su vida, por su vida misma. Las personas agradecidas tienden a sentir agradecimiento incluso cuando es claro que buena parte de la responsabilidad en la consecución de algo valorado, es de sí mismo. McCullough et al. (2002) ejemplifican con el caso de un competidor olímpico de natación que, al ganar la medalla de oro, agradece por el apoyo de su familia, la perseverancia y sabiduría de su entrenador, los agentes que financiaron su presencia en los juegos, aún siendo consciente de lo personal de su logro.

Por último, la faceta de densidad hace referencia al número y naturaleza de agentes externos hacia los cuales se agradece. Como se mencionó con anterioridad, los agentes de agradecimiento pueden clasificarse en interpersonales -las personas que benefician de algún modo; es este un elemento social- y transpersonales, aquellos agentes de la vida espiritual del ser humano a los que depositamos la capacidad de brindarnos dones o regalos, tales como Dios, el cosmos, la naturaleza, la vida misma.

McCullough et al. (2004) han hallado en investigación evidencia que apoya la hipótesis de la gratitud en el plano de los afectos intermedios, desarrollando un método de medición por el cual interroga a los sujetos de investigación hasta qué extensión se han sentido agradecidos durante el día -el estudio original, desarrollado para población anglosajona, contempla tres palabras sinónimas para evaluar el sentimiento de agradecimiento: grateful, thankful, appreciative. En español, no se registran multiplicidad de términos para la emoción de agradecimiento, lo cual contacta con la necesidad de estudiar los elementos culturales y cognitivos intervinientes en la percepción y comunicación de las emociones. En la investigación de referencia, se solicita a los participantes, posterior a la determinación del nivel de gratitud disposicional -entre otras medidas-, que lleven un registro diario en donde completan la pregunta detallada supra y las ocasiones en las que sienten agradecimiento por algún evento específico acontecido durante la jornada, debiendo puntualizar la intensidad de su sentimiento. Las personas que reportaban tener un afecto especialmente agradecido durante el día, percibían emociones de agradecimiento con mayor frecuencia e intensidad, siendo estos sujetos quienes puntuaban más alto en los índices de gratitud disposicional.

En relación con la interacción entre los tres niveles afectivos propuestos por Rosemberg (1998), no solamente destaca la organización jerárquica de los mismos, por los cuales el rasgo afectivo influye sobre el estado anímico o humor y éste, a su vez, facilita la percepción de emociones. Para McCullough, Tsang y Emmons (2004), entre otros autores, es importante la mediación de la gratitud como rasgo afectivo entre el estado anímico y la emoción. 
La hipótesis de la conductancia postula, que el rasgo afectivo facilitará la respuesta emocional ante un evento. Según esta hipótesis, un rasgo afectivo mediará positivamente entre el estado anímico y la respuesta emocional ante eventos, permitiendo preveer el estado anímico de un individuo consecuente a una reacción emocional en función del rasgo.

Por el contrario, la hipótesis de la resistencia postula que los rasgos afectivos no median entre el estado anímico y la emoción: en función de que una persona que tiende al agradecimiento no precisa de eventos específicos para sentirse agradecido, se supone que a mayor predisposición a la gratitud menor será la relación entre el estado anímico y los eventos que evocan gratitud.

Los resultados de las investigaciones efectuadas al respecto arrojan consistentes datos a favor de la hipótesis de la resistencia aplicada a la gratitud (McCullough, Tsang y Emmons, 2004), permitiendo aseverar que los individuos que por alguna razón están predispuestos a sentirse agradecidos no necesitan de eventos específicos en sus vidas diarias para percibir gratitud.

Este hecho apunta a que las personas con predisposición a la gratitud dirigen su atención hacia aquellos aspectos vitales positivos actuales o pasados que, al menos en algún aspecto, pueden ser atribuidos a la acción de un ente externo a sí mismo, de modo tal, que no requieren de la ocurrencia de un evento en particular para percibir agradecimiento. La gratitud disposicional, podemos hipotetizar entonces, posee de manera subyacente un esquema cognitivo que se caracterizaría por una valencia primordialmente positiva y benevolente del mundo (McCullough, Emmons y Tsang, 2002), mundo social y mundo interno, pudiendo considerar, sin embargo, los aspectos negativos de éstos, por lo que acentuar los eventos positivos en este contexto reviste un carácter de gratuidad (Moyano, 2009).

\section{Objetivos para la inclusión de la Gratitud en la psicoterapia}

Previo a la descripción de las intervenciones psicológicas ideadas para ayudar a las personas a percibir gratitud, es necesario diferenciar los objetivos con los que podrían aplicarse estas intervenciones en el marco de un proceso psicoterapéutico. Debido a la falta de investigación y desarrollo teórico en el área (McCullough, Emmons y Tsang, 2002; Vázquez et al, 2006; Moyano, 2009), se pueden analizar vías no exploradas para producir efectos positivos a nivel clínico en el marco de la psicoterapia, así como la potencialidad de la gratitud para facilitar el logro de objetivos psicoterapéuticos habituales. Dependiendo de la finalidad con que se decida incluir la gratitud en el proceso psicoterapéutico, deberían efectuarse modificaciones en los procedimientos técnicos de su implementación. 
Es posible plantear tres tipos de objetivos: la gratitud a nivel emocional por sí misma, la gratitud disposicional, y la gratitud como medio para alcanzar objetivos psicoterapéuticos tradicionales.

El desarrollo de la gratitud como emoción positiva y sus concomitantes efectos: se refiere a desarrollar gratitud estado. En función de los beneficios para la salud integral y el bienestar psicológico de las personas que se han documentado asociados a la gratitud, el facilitar la percepción de agradecimiento puede resultar un objetivo terapéutico en sí mismo dentro del proceso de psicoterapia. Fredrickson (2003) propuso que la gratitud en tanto emoción positiva posee las propiedades de ampliar la mente y facilitar la construcción de recursos internos para el afrontamiento de las exigencias de la vida. Vázquez et al. (2006) reseñan una lista de beneficios, documentados en la literatura experimental, de la percepción de emociones de corte positivo, tales como mayor indulgencia en los juicios hacia uno mismo y los demás, mejor tolerancia del dolor físico, mayores posibilidades de resistir ante las adversidades, etc. Bono y McCullough (2006) reseñan la capacidad de la gratitud para solidificar recursos de apoyo social, beneficiando el bienestar social de las personas y, desde allí, aumentar el bienestar psicológico de los individuos en tratamiento. Emmons y McCullough (2003) observaron beneficios sobre la satisfacción vital, el bienestar emocional, aumento de los afectos positivos, del optimismo hacia el futuro, de una intervención basada en contar aspectos positivos de la propia vida por las cuales sentirse agradecido.

Con la persecución de este objetivo, el acento de las intervenciones estaría en su efectividad para facilitar la percepción de la emoción gratitud. Al no perseguirse el fin de desarrollar gratitud en un nivel de mayor estabilidad temporal por medio del desarrollo de los procesamientos y los eventos cognitivos que facilitan la experimentación de agradecimiento, similar a los niveles disposicionales de gratitud, las intervenciones estarían destinadas a enseñar técnicas/ejercicios al paciente e indicar su utilización con una frecuencia estipulada para aumentar la frecuencia, densidad y spam en la percepción de agradecimiento. Con respecto a lo anteriormente dicho, es importante recordar, como lo plantea Vázquez et al. (2006) en reseñas de varias investigaciones sobre la eficacia de las intervenciones positivas, que la frecuencia con que se indica la realización de los ejercicios, aparece como una variable de influencia sobre la eficacia de los mismos para desarrollar bienestar. Vázquez puntualiza la necesidad de mayor investigación en este sentido (Vázquez et al, 2006).

Otro punto en contra de este objetivo es que, como señala Sheldon y Lyubomirsky (2006), existe evidencia de que el sostenimiento a largo plazo en la realización de un ejercicio para aumentar el nivel de bienestar subjetivo depende tanto de factores personales, tales como rasgos de personalidad, valores, motivaciones, necesidades, como de percepción de concordancia entre el ejercicio y las motivaciones e intereses personales del individuo al momento de comenzar a realizarlo. Desde este 
punto de vista, señalan los autores, las intervenciones no resultarán de agrado para todos los individuos, por lo que su eficacia puede verse disminuida si dependen de la aplicación a largo plazo (Sheldon y Lyubomirsky, 2006). En el apartado sobre las intervenciones psicológicas desarrolladas para propiciar vivencias de agradecimiento se analizarán los resultados de investigaciones realizadas por Lyubomirsky sobre las mismas y su potencialidad para sostenerse a largo plazo.

El desarrollo de disposición a la gratitud (gratitud rasgo): la gratitud disposicional se asocia positivamente a la satisfacción con la vida, emociones positivas, vitalidad y optimismo, aspectos prosociales como la empatía y el altruismo, la religiosidad y espiritualidad; y negativamente con la tendencia a sentir envidia, emociones negativas, ansiedad, depresión (McCullough et al., 2002). Por definición, la gratitud disposicional implica la tendencia a percibir gratitud con mayor frecuencia, intensidad, por más personas y por mayor cantidad de situaciones en aquellos que la poseen que en quienes no (McCullough et al., 2002). Varios autores (Lyubomirsky et al., 2005; Fredrickson, 2003; McCullough et al., 2002; Emmons, 2007) señalan que sentir agradecimiento con una elevada frecuencia e intensidad ayuda a las personas a saborear la vida, es decir, a aprovechar los elementos positivos de la existencia para sentir bienestar, en lugar de esperar el suceso de eventos especiales para lograr desarrollar emociones positivas. Desde este punto de vista, propender al desarrollo de la gratitud disposicional es un objetivo muy deseable. Lamentablemente, no se posee demasiada información sobre las condiciones que propician la gratitud como rasgo. La literatura experimental sobre las intervenciones psicológicas que ayudan a las personas a percibir agradecimiento como un estado o nivel emocional (Emmons y McCullough, 2003; Sheldon y Lyubomirsky, 2006, Seligman et al., 2005; Lyubomirsky, King y Diener, 2005) no explora si, como resultado de la aplicación de las mismas, se produce un aumento de la gratitud disposicional. La falta de modelos explicativos de la dinámica psicológica de la gratitud como un rasgo, dificulta la proposición de hipótesis sobre intervenciones que podrían resultar eficaces en este sentido o, si fuere factible, producir con intervenciones psicológicas dicho desarrollo. Si, tomando algunas hipótesis planteadas por Emmons y McCullough (McCullough, Emmons y Tsang, 2002) sobre la gratitud disposicional, haciendo eco de las posturas cognitivistas, las personas con tendencia al agradecimiento poseen un esquema cognitivo de ciertas características y un procesamiento cognitivo caracterizado por filtrajes de estilo positivo y atribuciones externas (McCullough et al, 2002), entonces es dable suponer que las intervenciones psicológicas utilizadas en el marco de las psicoterapias cognitivas, tales como el diálogo socrático, la retribución, la búsquedas de soluciones alternativas, la psicoeducación misma, pueden favorecer el desarrollo de la gratitud disposicional si se orienta el proceso hacia el estilo de 
procesamiento y las cogniciones que, como se analizó previamente, se asocian a la percepción del agradecimiento.

En este sentido, la similitud entre gratitud y optimismo, en tanto procesamiento cognitivo que resalta los aspectos positivos o benevolentes del mundo, mundo social, y mundo interno, uno hacia el pasado/presente y otro hacia el presente/futuro, permite suponer la factibilidad de diseñar dispositivos psicológicos para el desarrollo de la gratitud disposicional, como se ha desarrollado y comprobado su eficacia para el optimismo a través de la modificación de los estilos atributivos (Seligman, 2003; Seligman et al., 1995). De cualquier modo, se requiere mayor investigación en este punto.

Desarrollo de la gratitud como objetivo secundario a la modificación de esquemas cognitivos subyacentes y estilos de procesamiento cognitivo: por último, es posible plantearse la inclusión de la gratitud dentro de la psicoterapia cognitiva como un medio para alcanzar objetivos terapéuticos tradicionales, no directamente asociados con los beneficios documentados de la gratitud, como rasgo o como estado.

Como ya se mencionó, la literatura hace referencia a los aspectos cognitivos que propician la vivencia de gratitud (Emmons, 2007; Bono y McCullough, 2006). Las intervenciones desarrolladas para propiciar estados de agradecimiento implicarán el desarrollo de estas cogniciones específicas, facilitando un cambio cognitivo en ese sentido.

Independientemente de la relación con la gratitud disposicional, el desarrollo de las cogniciones descriptas en la literatura como propiciadoras de agradecimiento, implican un proceso cognitivo caracterizado por filtrajes positivos -en contraposición del filtraje negativo propio de los cuadros depresivos y ansiosos-, característica que la gratitud compartiría con el optimismo. Al colocar los elementos bondadosos, beneficiosos, protectores de la vida en primer plano, se ensaya la aplicación de un estilo de procesamiento alternativo a los que típicamente se hallan en los funcionamientos psicopatológicos disfóricos o ansiosos.

Además, la experiencia de gratitud implica la aplicación de un estilo atribucional, al menos en parte externo (McCullough, Emmons y Tsang, 2002), al percibir aspectos positivos no atribuibles a la acción de uno mismo; este patrón atribucional se relaciona de manera indirecta con los estilos atribucionales de los pacientes depresivos, en tanto éstos tienden a atribuir los fracasos y los perjuicios a la acción de uno mismo y los éxitos y beneficios a la acción externa (Beck et al., 1979). Esta característica de la gratitud implica que su utilización como herramienta para modificar estilos cognitivos debe ser cuidadosa y acompañada de otros recursos terapéuticos que permitan reforzar un autoconcepto positivo, de manera tal que se pueda agradecer a otros a la vez que se reconoce la propia responsabilidad en el logro de metas o la percepción de beneficios. La experiencia de agradecimiento implica necesariamente la consideración de un yo positivo (Moyano, 2009), como se mencionó con anterioridad, por lo que fomentarlas 
debería facilitar una mejoría en el autoconcepto, especialmente si este objetivo se ve abordado desde distintas técnicas.

El desarrollo de los procesamientos cognitivos descritos, facilitarían modificaciones en los esquemas cognitivos subyacentes (Beck et al., 1979; Meichembaum, 1988), en tanto iluminan aspectos determinados de la realidad favoreciendo un conocimiento diferencial del mundo, mundo social y mundo interno. El reconocer que se ha recibido algún tipo de beneficio, algo evaluado como positivo y valioso, proviniendo de alguna entidad externa a sí mismo, confirma la bondad del mundo externo y lo positivo del mundo interno en tanto objeto de beneficios.

Se considera entonces, que aplicar intervenciones para desarrollar gratitud aporta al logro de objetivos psicoterapéuticos clásicos de las psicoterapias cognitivas. Es posible que los terapeutas, desde modelos cognitivos u otros modelos teóricos, hayan implementados recursos terapéuticos relacionados con la gratitud, pudiendo a través de la línea de investigación actual en el marco de la Psicología Positiva sistematizar los conocimientos teóricos y calibrar las intervenciones clínicas en este sentido.

\section{Las intervenciones psicológicas}

Para abordar la descripción de las intervenciones psicológicas destinadas a facilitar experiencias de agradecimiento, dividiremos las mismas en técnicas específicas y técnicas tradicionales.

Las técnicas específicas son aquellas intervenciones desarrolladas en el marco de la Psicología Positiva con el objetivo puntual de facilitar la experimentación de agradecimiento. El desarrollo de las mismas es reciente y no pueden, por su naturaleza, ser aplicadas al desarrollo de otras emociones aunque, se hipotetiza, pueden aportar al logro de objetivos tradicionalmente perseguidos por las psicoterapias cognitivas.

En contraposición, se enumeran técnicas tradicionales, es decir, aquellas desarrolladas en el marco de las Psicoterapias Cognitivas para la modificación de las cogniciones, emociones y conductas problemas. Se analiza cómo estas intervenciones pueden ser aplicadas a la facilitación de experiencias de agradecimiento y al aumento de la gratitud en las personas.

Intervenciones específicamente desarrolladas para facilitar experiencias de gratitud

\section{Carta de Gratitud}

Seligman (2003) propone un ejercicio conductual denominado: Carta de Gratitud, en el cual se solicita a los participantes redactar una carta hacia una 
persona que lo haya beneficiado de algún modo, con la intención clara y explícita de entregar la misiva a su destinatario. Parte del ejercicio consiste en desarrollar un espacio para la entrega o la lectura de la carta junto a su destinatario. Seligman aplicó esta técnica en su clase de Psicología Positiva en la Universidad de Pensilvania, recogiendo experiencias altamente emotivas y placenteras para los participantes, demostrando el poder movilizador de esta técnica.

En el 2005, Seligman llevó adelante una investigación por asignación aleatoria a diversas condiciones experimentales, dentro de las cuales se encuentra la mencionada intervención. Se observó que la carta de gratitud produjo aumentos en los niveles de bienestar subjetivo al momento de concluir con la aplicación del ejercicio, pero que sus efectos positivos se vieron neutralizados (en seguimiento) a los seis meses.

Por otra parte, este mismo ejercicio fue incluido en la diagramación de una psicoterapia positiva, junto a los otros cuatro ejercicios que formaron parte de la mencionada investigación (Seligman et al., 2005) en un protocolo manualizado de doce sesiones. Se observó que, en comparación experimental con una terapia convencional y con terapia convencional sumada a medicación, la intervención positiva fue más eficaz a la hora de disminuir la sintomatología depresiva.

Estos resultados preliminares indican que el ejercicio de la carta de gratitud posee potenciales beneficios para el tratamiento de cuadros psicopatológicos, especialmente a la hora de mejorar los niveles de emociones positivas. Sin embargo, la dinámica por la cual ejerce influencias sobre el funcionamiento psicológico debe ser esclarecida, debido a que sus efectos son limitados en el tiempo y requieren de intervenciones adicionales para asociarse a los efectos deseados.

Analizando las características de la intervención, podemos suponer que, debido al efecto altamente activador de este ejercicio, tal como lo describe Seligman en la aplicación del mismo que realizó en su clase sobre Psicología Positiva -aplicación documentada en la publicación La Auténtica Felicidad (2003)- escribir y leer una carta de gratitud moviliza el reconocimiento sobre el poder del agradecimiento, sobre las emociones, pero no genera una modificación en las construcciones cognitivas subyacentes que permitan sostener el aumento de emociones positivas en el tiempo, al menos no por sí sola. En función de las características mismas de la intervención, además, es poco probable que esta pueda indicarse como un ejercicio frecuente a lo largo del tiempo.

De esto se deriva, retomado el análisis de los objetivos con los que puede incluirse la gratitud en las Psicoterapias Cognitivas, que, claramente, escribir y entregar una carta de agradecimiento contribuye de manera directa al primer objetivo planteado: brindar estímulos para el desarrollo de la emoción de agradecimiento. Para que este ejercicio aporte a los demás objetivos, es decir, desarrollar gratitud disposicional y facilitar la modificación de esquemas cognitivos subyacentes y procesos cognitivos, se deberán adherir otras medidas a ésta. 
Desde este punto de vista, se considera que es posible incluir el ejercicio de la carta de gratitud en la psicoterapia cognitiva conductual a modo de un experimento conductual. Desde este punto de vista, la intervención tendría por objetivo descubrir la potencialidad del agradecimiento para aumentar los niveles de bienestar, facilitando el desarrollo de la motivación para realizar conductas de agradecimiento de manera frecuente. Además, se propondría la finalidad de descubrir el impacto emocional que provoca en el otro a quien se agradece, mostrando claramente el beneficio de acercamiento emocional con aquellos a los que demostramos abiertamente nuestro agradecimiento.

El experimento conductual así llevado a cabo podrá enfatizar ciertos aspectos bondadosos del mundo social, en tanto adquiero clara conciencia en el aquí y ahora de que alguien me ha ayudado de alguna manera, y del mundo interno, en tanto he sido objeto de la consideración de alguien a quien, además, le interesa mi respuesta de agradecimiento.

Este episodio movilizador deberá ser utilizado en el marco de la psicoterapia cognitiva, mediante las técnicas típicamente utilizadas, para construir a partir de la evidencia que aporte un camino hacia la consideración más sistemática de los beneficios recibidos y del valor que los mismos poseen, en tanto podrían no haber sido experimentados.

\section{Contar bendiciones}

Este ejercicio, diseñado inicialmente por Emmons y McCullough (2003) para poner a prueba la hipótesis que la gratitud desarrolla emociones positivas, disminuye las negativas, y aumenta los niveles de bienestar subjetivo, psicológico y físico, consta de una premisa básica y diversas versiones sobre la frecuencia y modalidad de realización (Vazquez et al., 2006) .

La premisa básica consiste en dirigir la atención hacia aquellos aspectos de la vida por los que puedo sentir agradecimiento. Sobre esta premisa, algunos autores plantean tareas escritas, como Emmons et al. (2003) y Seligman et al. (2005). Otros, como Sheldon y Lyubomirsky (2006) plantean una consigna que articula escritura en una primera etapa con sólo pensamiento de manera posterior. Por otra parte, se plantea distintas frecuencias para la realización del ejercicio: en algunos casos, se solicita llenar un formulario con cinco cosas buenas que ocurrieron en la semana anterior McCullough et al. (2003), en otros casos, se solicita agradecer por cosas buenas una o tres veces a la semana (Seligman, 2005). En el Diario de Gratitud sugerido por Robert Emmons en el libro Gracias (2007) se plantea continuar un diario en el que cada día se registren los aspectos positivos por los cuales estar agradecido. Por último, existen diferencias en cuanto a la percepción de agradecimiento 
y la prescripción de dar las gracias, es decir, entre los elementos cognitivo/ afectivos de la gratitud y el componente conductual.

De los formatos estudiados, los que obtuvieron resultados positivos en el objetivo de aumentar el nivel de bienestar, son los planteados por Emmons y McCullough en su versión de contar bendiciones una vez al día durante dos o tres semanas. En algunos estudios, los efectos positivos se registraron al terminar la aplicación de la técnica (Emmons et al., 2003) y en otros los efectos positivos se observaron no al terminar la aplicación, sino en el seguimiento a seis meses (Vázquez et al., 2006).

Los resultados contradictorios en cuanto a la efectividad de esta intervención pone de manifiesto la presencia de limitaciones en el modo en que se aplica el desarrollo de emociones de agradecimiento al intento de modificar el estado anímico de los individuos. Pone el acento además en la necesidad de investigar con mayor profundidad los mecanismos subyacentes que se juegan al momento de realizar las intervenciones, mecanismos que seguramente se conjugan para desarrollar diferentes resultados. Sin embargo, al igual que con la carta de gratitud, el ejercicio de contar bendiciones se incorporó en un modelo de psicoterapia positiva aplicada con éxito a pacientes con síntomas depresivos, lo cual indica que, más allá del desconocimiento sobre los mecanismos de acción sobre el funcionamiento psicológico, las intervenciones psicológicas que buscan desarrollar la gratitud son eficientes y dignos de ser tenidos en cuenta en el tratamiento de los trastornos emocionales.

Se analiza que, al solicitar a un individuo que se enfoque en aquellos aspectos bondadosos por los que puede estar agradecido, se lo influencia para que efectúe de manera sistemática un procesamiento cognitivo de filtraje positivo, con lo que este ejercicio aporta a los objetivos terapéuticos de la psicoterapia cognitiva comportamental. Debido a que es eficiente para elicitar emociones positivas de agradecimiento, se considera que aporta al objetivo de mejorar el estado anímico del paciente y las consecuencias asociadas.

Por otra parte, al estar aportando información de manera sistemática sobre la existencia de numerosos factores positivos en la vida otorgados por algún agente externo, se aporta a desarrollar modificaciones en el esquema cognitivo subyacente sobre el mundo, mundo social y yo, permitiendo arrojar luz sobre el hecho que la vida está repleta de aspectos bondadosos que podrían no haber estado allí.

Otras posibles intervenciones cognitivas para el desarrollo de gratitud y el aprovechamiento de sus beneficios en el logro de los objetivos terapéuticos

\section{Psicoeducación}

El libro de Robert Emmons, Gracias: de cómo la gratitud puede hacerte feliz, publicado en el año 2007, trabaja en un lenguaje sencillo y efectivo el concepto de 
gratitud, los beneficios asociados a la misma y algunas técnicas simples para lograr ser más agradecidos. Esta publicación ofrece criterios claros y comprensibles de por qué es positivo intentar ser más gratos en nuestra cotidianeidad.

Al informar sobre la dinámica y efectos de la gratitud se puede mejorar la motivación para producir cambios en ese sentido y ayudar al paciente a comprender aspectos del funcionamiento psicológico saludable.

Desde este punto de vista, la psicoeducación en gratitud se convertiría en una herramienta valiosa para lograr la inclusión exitosa de la gratitud en la psicoterapia, en el marco de los objetivos propuestos para dicha integración.

\section{Aprendizaje Vicario: el terapeuta como modelo de agradecimiento}

Las conceptualizaciones de Albert Bandura (1987) sobre el aprendizaje vicario señalan que los seres humanos no solamente aprendemos por ensayoerror o por razonamiento: los modelos de conducta a los que tenemos acceso son una fuente de gran importancia para el desarrollo de los patrones conductuales.

Haciendo eco de este concepto, el terapeuta, desde las líneas cognitivas de tratamiento, puede ser pensado como un modelo de aprendizaje para el paciente, especialmente cuando se han logrado establecer ciertas condiciones vinculares de confianza, empatía y colaboración. En este sentido, la conducta del terapeuta funciona como modelo para la adquisición de ciertos comportamientos, teniéndose en cuenta las características que debe poseer el modelo. En este sentido, se señala que los modelos que comparten ciertas características con el observador resultan más efectivos a la hora de facilitar el aprendizaje vicario (Bandura, 1987): si bien los modelos exitosos son imitados en sus conductas debido al reforzamiento que implica el resultado positivo obtenido por los mismos, la observación de este tipo de modelo favorece el desarrollo de planes a largo plazo. De cualquier modo, los modelos disímiles pueden asociarse con el aprendizaje vicario de conductas (Bandura, 1987). Otras características del modelo que favorecen el aprendizaje vicario son el carácter afectivo y reforzante o el estatus social del mismo (Gutiérrez Maldonado, 2004; Zaldivar Perez, 1994).

La psicoterapia cognitiva puede valerse del recurso del aprendizaje vicario para facilitar el desarrollo de elementos disposicionales hacia la gratitud, en la medida en que el terapeuta pueda manifestar agradecimiento y acompañe a la persona en tratamiento a que agradezca por sus beneficios y logros.

El que el terapeuta mismo exprese gratitud por aspectos personales, explique el porqué se considera agradecido, resaltando las cogniciones que caracterizan la experiencia de gratitud -reconocimiento de algo positivo, reconocimiento del valor de lo recibido, reconocimiento de que, en función de no ser controlado por uno mismo, el beneficio valorado podría no haber sido recibido- e inste al paciente a 
valorar qué cosas podría agradecer de su propia experiencia facilitaría el desarrollo de motivación para realizar un esfuerzo personal en la búsqueda de la experiencia de agradecimiento y brindaría elementos claros de cómo se puede ser agradecido.

Esto implicaría que el terapeuta exprese gratitud con cierta frecuencia, incluso hacia el paciente mismo. Actos que son considerados habituales y esperados por el paciente pueden ser reconocidos como dignos de agradecimiento, a la vez que habilita al pedido del terapeuta de agradecer en ciertas ocasiones.

Por otra parte, las conductas de agradecimiento efectuadas por el terapeuta implican un reforzamiento al desarrollar emociones placenteras en el paciente, sirviendo de claro ejemplo sobre los beneficios interpersonales de expresar gratitud. El terapeuta podría utilizar anécdotas sobre la expresión o experimentación de emociones de agradecimiento en las que se remarque el beneficio obtenido, de modo que se vea reforzada la conducta deseable.

\section{Diálogo socrático}

Mediante preguntas el terapeuta puede ayudar a la persona a descubrir en un evento ordinario o posesión actual las características de un aspecto para sentir agradecimiento. Mediante la formulación de preguntas como:

¿Es posible que este evento, que concluyó como me mencionas, haya concluido de un modo negativo?

¿Qué consecuencias tendría para tu vida la ausencia/presencia de este aspecto?

$¿$ Es posible que este aspecto pueda faltar en tu vida? ¿Conoces casos en que ha sido así? - $\mathrm{O}$ en cuyo caso, su inversa de tratarse de un aspecto negativo cuya ausencia queremos agradecer, como podría ser violencia familiar, accidentes de gravedad, lesiones o limitaciones físicas, etc.-,

¿Estamos en condiciones de afirmar que puedes estar agradecido por esto?

De ser así, ¿A quién agradecerías? ¿A alguien en particular, a Dios, a la vida?

De esta manera, podemos ir facilitando el direccionamiento del foco de atención del paciente hacia el reconocimiento de:

1) aspectos positivos

2) el valor de los mismos para la vida

3) la posibilidad de ausencia de tales aspectos

4) la intervención de un agente externo a sí mismo

\section{Técnicas de relajación}

Las técnicas de relajación son tradicionalmente aplicadas para facilitar el manejo de la ansiedad y mejorar la calidad de vida de las personas (Meichembaum, 1988; Labiano, 2004; Carr, 2004; Gutiérrez Maldonado, 2004). 
Bono y McCullough (2006) citan investigaciones en las que se ha observado que la aplicación de técnicas de relajación muscular progresiva, meditación e imaginería facilita la percepción de estados de agradecimiento.

El facilitar la experiencia de gratitud puede permitir a la persona reconocer el aumento de satisfacción y de la afectividad positiva asociada a la misma, a partir de la cual se puede acrecentar la motivación para realizar ejercicios y actividades específicas para el desarrollo de mayores niveles de gratitud.

\section{Conclusiones}

A partir de los argumentos expuestos, podemos concluir que el desarrollo de gratitud es un objetivo deseable en el marco de los procesos psicoterapéuticos, debido a los beneficios bio-psico-sociales que se asocian a su experimentación (Bono y McCullough, 2006).

Hasta el momento, se han propuestos y estudiado ejercicios que pueden facilitar la percepción de estados de agradecimiento, observándose que tal experiencia disminuye la sintomatología depresiva (Seligman et al, 2005), por ejemplo, y aumenta la satisfacción vital (Seligman, 2003) y, por tanto, el bienestar subjetivo. De estos resultados, deriva que la variedad de intervenciones diseñadas para provocar vivencias de gratitud resultan adecuadas a los fines de la psicoterapia y se asocian a los resultados para los que fueran diseñados (Bono y McCullough, 2006).

Los protocolos desarrollados para el tratamiento de la depresión desde la perspectiva de la Psicología Positiva incluyen de manera protagónica las intervenciones asociadas a la experiencia de gratitud, junto al descubrimiento y desarrollo de las fortalezas personales (Seligman et al., 2005; Vázquez et al., 2006). Estos programas han demostrado poseer adecuados niveles de eficacia, con cuyos resultados se acentúa la importancia de la inclusión de la gratitud en los procedimientos psicoterapéuticos.

A partir de los elementos abordados, es posible pensar el desarrollo de gratitud como un objetivo posible, es decir, que puede ser alcanzado a través de las intervenciones específicamente desarrolladas a tales fines como a través de técnicas tradicionales en el marco de las psicoterapias cognitivo comportamentales (Bono y McCullough, 2006).

De todos modos, y a pesar de que el desarrollo de gratitud en la psicoterapia cognitiva es compatible con los objetivos tradicionalmente trazados para tales procesos terapéuticos, el desarrollo de los modelos teóricos y las intervenciones asociadas se encuentran en estado de construcción, detectándose lagunas de conocimiento que resultan de relevancia para una delimitación clara de los 
alcances de las intervenciones, los objetivos con los que se buscará la inclusión de la gratitud en las terapias cognitivas, y los procedimientos que habrá de seguir dicha inclusión. Acentuar la importancia de continuar investigando estos factores se convierte en imprescindible, entonces, en función del impacto que las vivencias de gratitud poseen para el bienestar psicofísico y social de las personas.

\section{Referencias Bibliográficas}

Bandura, A. (1987). Pensamiento y acción. Fundamentos sociales. España: Martínez Roca.

Beck, A.T.; Rush, A.J.; Shaw, B.F. \& Emery, G. (1979). Terapia Cognitiva de la Depresión. $5^{\circ}$ Edición. España: Desclee de Brouwer.

Bono, G. \& McCullough, M.E. (2006). Positive responses to benefit and harm. Bringing forgiveness and gratitude into Cognitive Psychotherapy. Journal of Cognitive Psychotherapy: an international quarterly, 20, 147-158.

Carr, A. (2004). Psicología Positiva. La ciencia de la felicidad. España: Paidós.

Emmons, R. A., \& McCullough, M. E. (2003). Counting blessings versus burdens: An experimental investigation of gratitude and subjective wellbeing in daily life. Journal of Personality and Social Psychology, 84, 377-389.

Emmons, R. A., \& McCullough, M. E. (2003b; Eds.), The psychology of gratitude. New York: Oxford.

Emmons, R.A. (2007) ¡ Gracias! De cómo la gratitud puede hacerte feliz. España: Ediciones B.

Fredrickson, B.L. (2003). Gratitude, like other positive emotion, broadens and builds. En Emmons R.A. \& McCullough M.E. The psychology of gratitude, 145-166. New York: Oxford.

Gutierrez Maldonado, J. (2004). Ansiedad y salud. En Oblitas, L.A. La Psicología de la Salud y Calidad de Vida. México: Thompson Learning.

Labiano, M. (2004). Estrategias de Mejoramiento de la Calidad de Vida. En Oblitas, L. A. Psicología de la Salud y Calidad de Vida. México: Thompson Learning.

Lyubomirsky, S., King, L. \& Diener, E. (2005). The benefits of frequent positive affect: does happiness lead to success? Psychological Bulletin, 131, 803-855.

McCullough, M. E., Kilpatrick, S. D., Emmons, R. A., \& Larson, D. B. (2001). Is gratitude a moral aaffect? Psychological Bulletin, 127, 249-266. 
McCullough, M. E., Emmons, R. A., \& Tsang, J. (2002). The grateful disposition: A conceptual and empirical topography. Journal of Personality and Social Psychology, 82, 112-127.

McCullough, M.E., Tsang, J., \& Emmons, R.A. (2004). Gratitude in Intermediate affective terrain: links of grateful moods to individuals' differences and daily emotional experience. Journal of Personality and Social Psychology, 86, 377-389.

Meichembaum, D. (1988). Terapias Cognitivas-conductuales. En Linn, S.J. \& Garske, J.P. (comp.). Psicoterapias Contemporáneas (pp.331-362). España: Desclee de Brouwer.

Moyano, N. C. (2009). Gratitud en la Psicología Positiva. Psicodebate. Psicología, Cultura y Sociedad, Vol. 10, pp. 103-117.

Peterson, C. \& Seligman, M.P. (2004). Character Strengths and Virtues: a handbook and classification. Washington D.C.: American Psychology Association.

Rosenberg, E. L. (1998). Levels of analysis and the organization of affect. Review of General Psychology, 2, 247-270.

Seligman, M. E. P., Reivich, K., Jaycox, L. y Gillham, J. (1995). The optimistic child. A proven program to safeguard children against depression and build lifelong resilience. Nueva York: Harper Collins.

Seligman, M. P. (2003). La Auténtica Felicidad. $1^{\circ}$ Edición. España: Vergara.

Seligman, M. E. P., Steen, T. A., Park, N. y Peterson, C. (2005). Positive Psychology progress: Empirical validation of interventions. American Psychologist, 60, 410-421.

Sheldon, K.M., \& Lyubomirsky, S. (2006). How to increase and sustain positive emotions: The effects of expressing gratitude and visualizing best possible selves. The Journal of Positive Psychology, 1(2), 73-82.

Vazquez, C.; Hervas, G., Ho, S. (2006). Intervenciones clínicas basadas en la Psicología Positiva: fundamentos y aplicaciones. Psicología Conductual, 14 (3), pp. 401-433.

Zaldivar Pérez, D.F. (1994). Entrenamiento asertivo, Aprendizaje social y Entrenamiento en Habilidades Sociales. Revista Cubana de Psicología, Vol. 12, (2), pp. 99-107. 